\title{
Condition Based Maintenance of Grid Equipment and Its Prospect Based on "Internet +"
}

\author{
Ziqi Wang
}

North China Electric Power University, No.2 Beinong Road, Changping District, Beijing, China

axxdwzq@163.com

\begin{abstract}
With the rapid development of power grid technology, production and living have put forward higher and higher requirements for the safety, stability and economic operation of power system. The past Plan-Maintenance mode cannot meet the needs of power grid development, and Condition Based Maintenance has become an inevitable trend. To ensure the safety and reliability of the equipment is the key to the electric power enterprise to adapt to the current power development requirements and market competition pattern. After consulting a large amount of data, the paper finds out the key factors that affect the development of the equipment status monitoring of power grid, and puts forward some rectification proposal. This paper proposes to build a cloud platform for information sharing in Beijing by using the Internet technology, which can realize accurate state evaluation and risk prediction, and reduce maintenance costs and improve power safety and reliability.
\end{abstract}

\section{Keywords: Condition Based Maintenance, Management Optimization, "Internet +"}

\section{Introduction}

Electric power industry is one of the most important energy industries in modern society, and the maintenance system of power grid equipment is also evolving correspondingly. The maintenance system of power grid equipment has changed greatly in different periods of industrial development. Overall, the evolution of power grid equipment maintenance system can be divided into three stages, Breakdown Maintenance, Time Based Maintenance, Condition Based Maintenance. [1]The evolution process is shown in Fig. 1.

\begin{tabular}{|l|l|l|}
\cline { 2 - 3 } $\begin{array}{l}\text { Breakdown } \\
\text { Maintenance }\end{array}$ & $\begin{array}{l}\text { Time Based } \\
\text { Maintenance }\end{array}$ & $\begin{array}{l}\text { Condition Based } \\
\text { Maintenance }\end{array}$ \\
\hline $\begin{array}{l}\text { Only if the } \\
\text { equipment is } \\
\text { out of order } \\
\text { can it be } \\
\text { repaired. }\end{array}$ & $\begin{array}{l}\text { Periodic } \\
\text { Maintenance } \\
\text { Rise and } \\
\text { develop after } \\
\text { Prior to 1950, } \\
\text { this approach } \\
\text { was widely } \\
\text { adopted in all } \\
\text { countries. }\end{array}$ & $\begin{array}{l}\text { Reliability } \\
\text { centered and a } \\
\text { combination } \\
\text { of multiple } \\
\text { technologies. } \\
\text { It was } \\
\text { developed } \\
\text { around 1980 } \\
\text { and has been } \\
\text { developed so } \\
\text { far. }\end{array}$ \\
& & \\
\hline
\end{tabular}

Fig. 1 Evolution course of power grid equipment maintenance system

Condition Based Maintenance, which is called Predictive Diagnostic Maintenance, is a comprehensive maintenance. This kind of maintenance method has great advantages compared with the Breakdown Maintenance and Time Based Maintenance. According to the current actual equipment working state, the use of preventive test, online monitoring information, condition 
evaluation of similar equipment defects, history information and other aspects of the data, Condition Based Maintenance can make a judgment on the parts of equipment failure and the development trend of fault degree. And then to security, reliability, environment, cost-based, through risk assessment, maintenance decision-making and other technical means to develop a scientific and effective maintenance program to ensure that the grid equipment can work in the best condition, which is a dynamic optimization process. Condition Based Maintenance not only improves the utilization of equipment, but also reduces the cost of equipment maintenance, is the most advanced maintenance.

\section{Substation Equipment Maintenance, Management Analysis and Optimization}

\subsection{Status quo of substation equipment maintenance}

At present, the following problems exist in the substation equipment:

(1) There are still a few of the old equipment running in the power system, and its equipment performance is poor, which also has more failures. After equipment maintenance, sometimes equipment performance has declined on the contrary.

(2) $10 \mathrm{kV}-35 \mathrm{kV}$ medium voltage equipment has obviously more defects. Due to the poor manufacturing process of some manufacturers and the improper selection of materials, the defects of the breaker mechanism are prominent. These defects become the major problems affecting the safe operation of the equipment, and the probability of repeated occurrence of them is higher.

(3) The overall operation level of transformer is improved, but the auxiliary equipment is still more defective.

(4)Some external reasons cause short-circuit fault. It not only increases the workload of maintenance, but also seriously threatens the safe operation of the system.

\subsection{SWOT Analysis of Substation Equipment Maintenance Management}

(1) Superiority Analysis

Most power supply companies have accumulated some experience, and its technical level and organizational capacity can meet the requirements of making a correct assessment on the equipment status.

(2)Inferiority Analysis

To carry out state maintenance, power supply companies is still in the exploratory stage, there are deficiencies, including:

1) The maintenance plan is not detailed enough.

2) There is no definite administration.

3) The skill level of maintenance personnel should be improved.

(3)Chance Analysis

Condition based maintenance of power transmission and transformation equipment has become one of the most important measures to optimize the production system management of power supply enterprises, and also become an important measure to ensure the safe, economic and stable operation of power grid and improve the reliability of power supply.

(4) Threat Analysis

The purchasing centralization of power supply companies leads to a decrease in the quality of power grid equipment and has a negative impact on state maintenance.

\subsection{Condition Based Maintenance Management Optimization}

At present, the power supply company has not formed the management process for the condition 
based maintenance of the power grid equipment. This paper will build the management process of the condition based maintenance of the power supply company transformer equipment, as shown in Fig. 2. It includes seven parts: status information collection, status evaluation, risk assessment, maintenance decision, maintenance plan, maintenance implementation and performance evaluation.

(1)Status Information Collection

The status information collection should include the data of the equipment's health status during the life cycle of the equipment. According to the production process, it can be divided into pre operation information, operation information, maintenance test information, and family defect information.

(2) Status Evaluation

The equipment state evaluation is in accordance with the technical standard of Beijing, through the collection of the equipment condition parameters to analyze and conclude the development trend of equipment. It includes equipment periodic evaluation and dynamic evaluation of equipment.

The equipment periodic evaluation is divided into three levels, evaluation of groups, evaluation of work areas and evaluation of Bureau.

Evaluation of Groups: According to the collection of equipment state information, the groups form the preliminary opinions about state evaluation, and report to the corresponding work area.

Evaluation of Work Areas: Work Area organizes professional person review the preliminary opinions, and put forward maintenance recommendations to form the work area report, then submits it to maintenance expert group.

Evaluation of Bureau: Maintenance expert group reviews the work area report and form the Condition based maintenance report finally.

- Equipment Status Information Collection

Fig. 2 Status Management Process 


\section{2) Dynamic Evaluation of Equipment}

It includes the assessment of the completion of the maintenance, the equipment to withstand bad work conditions and the evaluation of the new release of family defects, etc.

(3) Risk Assessment

Risk assessment and equipment periodic evaluation are carried out at the same time. Risk assessment should be carried out in accordance with the rules, combined with equipment status evaluation results, comprehensive consideration of risk of safety, economic and social impact, then determine the degree of risk.

(4)Maintenance Decision

The maintenance decision should be based on the results of the equipment status evaluation, refer to the risk assessment results, consider the requirements of power grid development, technical update and so on, comprehensive control center and safety inspection quality department opinions, according to technical standards to determine the maintenance category, maintenance items and maintenance time and so on.

(5)Maintenance Plan

The status maintenance plan shall be arranged in accordance with the maintenance procedures, various equipment status evaluation guidelines, equipment status evaluation results and so on.

(6)Maintenance Implementation

The implementation of maintenance is the concrete execution link of condition based maintenance, and the on-site condition based maintenance should be carried out according to the power failure maintenance plan issued and the relevant condition maintenance instruction.

(7)Performance Evaluation

Performance evaluation work can find out the main problems in the process of status maintenance work, and realize the dynamic management and continuous improvement of condition based maintenance work.

\section{Outlook based on Internet technology}

\subsection{Background}

At present, the accuracy and effectiveness of operation and maintenance of power grid equipment are far from meeting the requirements of the state maintenance of smart grid, and there are many technical bottlenecks:

First of all, the data format of the state information of power grid equipment is not unified and standardized, and the storage medium has different forms, which is not convenient for unified collection and management, so it is difficult to effectively accumulate and use;

Secondly, the status data size is larger, but currently on the market a variety of state detection device data storage capacity is limited, it is difficult to achieve large-scale storage and processing of the test data, a large number of valuable historical data are often discarded;

Last but not the least, it is difficult to achieve real-time sharing of information between the state detection device manufacturers and user units. Due to the limitation of information integrity, there is no deep level data mining between the state information of power grid equipment, so it is difficult to achieve accurate state evaluation and risk prediction.

\subsection{Application Principle and Implementation Steps}

The deep integration of Internet technology and traditional power grid equipment detection technology [2]can make the traditional grid equipment and its condition based maintenance products upgrade for Cloud Applications. Through the research based on cloud storage, a new model of smart grid equipment state maintenance of cloud computing and big data can provide new platform for equipment condition maintenance decision-making service for power units, in order to improve the accuracy of power equipment fault diagnosis, condition assessment and risk prediction.

The implementation step is divided into three steps. The first step is the collection of power equipment status information, the second step is the transmission of power equipment status information, and the third step is the processing of power equipment status information. (The 
specific process is shown in Fig. 3)

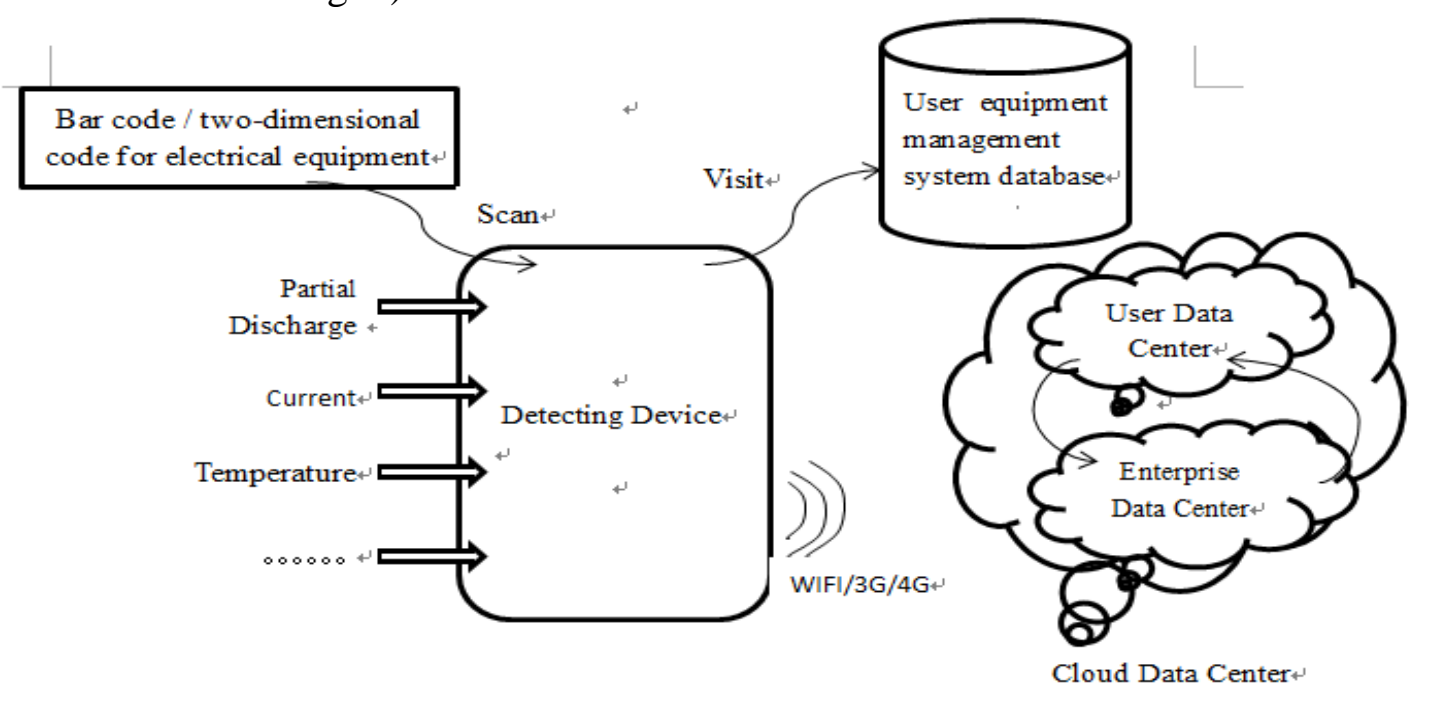

\subsection{Superiority[3]}

Fig. 3 "Internet+" Implementation Steps

(1) Reduce Maintenance Costs and Bring Economic Benefits

1) The construction of cloud platform is low cost and quick operation

In the traditional mode, the power enterprises need to invest a lot of money to purchase hardware equipment, build an entity platform to supervise the power equipment. In the cloud storage mode, the power companies do not need to invest additional funds to build the platform in addition to configuring the necessary terminal equipment to receive and storage services, only by the number of power users and power cycles, cloud services can be provided. In this way, it not only avoids the one-time investment risk of the power enterprise, reduces the use cost, but also can immediately use select the service that the electric power users need, which is convenient and quick. The real-time monitoring of the cloud platform can improve the accuracy of the judgment of power equipment operating conditions, thus saving a great deal of maintenance costs

2) Impetus to Social and Economic Development

Taking Beijing as an example, through the upgrade for the cloud applications of traditional power grid equipment and state detection products, it will create billions of yuan in market capacity conservatively, which will vigorously promote the development of Beijing smart grid monitoring industry and is expected to create billions of yuan of output value, nearly 10 million yuan tax in the next 3 5 years. At the same time, at least hundreds of employment opportunities for Beijing are to create. Due to the application of this technology will improve the reliability of power supply and bring down operation cost, so the social value will be very significant.

(2)Improve the Reliability of Power Supply

"Internet+" based on large data cloud computing, and it can approximate the operation rules of the grid equipment, reasonable arrangements for power grid equipment running time, extend the life of power equipment, improve power supply reliability.

(3)Optimize the internal human resources allocation of power enterprises

In the "Internet+" mode, maintenance and updating of the system are completed by cloud service providers, power companies can enjoy the latest and professional services at the lowest cost. It can promote the internal distribution structure optimization of power enterprises, and improve the personnel utilization and management rate in power enterprises.

(4) High Time Efficiency

Power equipment monitoring based on "Internet+" technology can provide fast query mode. Cloud platform operation can greatly improve the efficiency of access time and processing time efficiency, so that the power equipment in real-time and efficient monitoring by electric power enterprises and the smart grid comprehensive promotion feasibility is greatly improved.

(5) Large Data Storage Capacity 
The power equipment monitoring based on cloud platform can greatly improve the data storage capacity of power equipment, and meet the requirements of the current power system for data storage.

(6)Realize the Security Sharing of Data

The monitoring of power equipment based on Internet technology implements a privacy preserving and extensible cloud data sharing scheme, which can effectively protect the security of data in the process of storage and sharing.

References:

[1] J.H. Huang and L.S. Quan. Status quo and development of equipment condition based maintenance in high voltage power grid [J]. Power System Automation,2001,(16):56-61. (In Chinese)

[2] H.J. Wang. Construction of accounting large data analysis platform based on Cloud Computing [D]. Shanxi Finance and Economics University,2015. (In Chinese)

[3] M.J. Guo. Application Research of large data and cloud computing platform [J]. Modern Telecommunication Technology,2014,(08):7-11+16. (In Chinese) 